\title{
Análisis del golpeo de fondo en jóvenes jugadores de tenis en una competición modificada mediante sensores inteligentes
}

Groundstrokes analysis in a modified competition

in young tennis players through smart sensors

\author{
José Maria Giménez-Egido (i) \\ Departamento de Actividad Física y Deporte, Facultad de Ciencias del Deporte, Universidad de Murcia, España \\ josemaria.gimenez@um.es
}

Ángel Iván Fernández García

Departamento de Fisiatría y Enfermería, Facultad de Ciencias de la Actividad Física y del Deporte, Universidad de Zaragoza, Huesca, España angelivanfg@unizar.es

Alvaro Castellanos

Departamento de didáctica de la expresión musical plástica y corporal, Facultad de Humanidades y Ciencias de Educación, Universidad de Jaén, Jaén, España

acf00029@ujaen.es

\begin{abstract}
Resumen
La utilización de dispositivos inteligentes para observar el efecto de la modificación de reglas y equipamientos deportivos sobre las conducta técnico-táctica en etapas de formación, abre un nuevo campo de estudio en la mejora de los procesos de enseñanza-aprendizaje. El objetivo de estudio es utilizar el sensor inteligente "Zepp Tennis 2.2.1", para conocer el impacto de una competición modificada (altura de la red=0,80m y dimensiones del campo=18,00x8,23m) en jugadores de tenis sub-10, atendiendo a los criterios de optimización de las enseñanzas comprensivas. La unidad de análisis fueron los golpeos ejecutados ( $n=7758)$ en 40 partidos por 20 jugadores (edad media= 9.46 \pm 0.66 años). Las variables de estudio son: a) número de golpeos; b) porcentaje de golpes que impactan en el punto dulce de la raqueta; c) velocidad de bola; y d) revoluciones por minuto de la pelota. Los resultados más destacables son: a) un aumento del uso de golpeos planos sobre los de efecto liftado y cortado; y b) escasa aparición en juego del revés plano y cortado, aunque muestren mejores valores de velocidad lineal y rotacional que los golpeos de derecha. La reducción de la altura de la red y las dimensiones del campo inducen a los jóvenes jugadores a una conducta ofensiva de juego. Este comportamiento ofensivo, coincide con las recomendaciones que promueven las enseñanzas comprensivas sobre un proceso de enseñanza-aprendizaje óptimo.
\end{abstract}

Palabras claves: enseñanzas comprensivas, etapa de formación, rediseño competitivo, dispositivos inteligentes.

\begin{abstract}
Use of smart devices to show the effect of modification rules and sports equipment on technical-tactical behavior in training stages, opens a new field of study to improve the teaching-learning processes. The objective of the study is to use the smart sensor "Zepp Tennis 2.2.1", to know the impact of a modified competition (net height $=0.80 \mathrm{~m}$ and field dimensions $=18.00 \times 8.23 \mathrm{~m})$ in under-10 tennis players, according to the comprehensive approach criteria of optimization. The hits $(\mathrm{n}=$ 7758 ) during 40 matches played by 20 players (average age $=9.46 \pm 0.66$ years) were the unit analysis. The study variables were as follows; a) number of strokes, b) percentage of hits impact in sweet spot racket; c) ball speed; and d) ball spin. The results show as follow: a) an increase in the use of flat strokes over topspin and slice; and b) scarce flat and slice play of the flat backhand appearance, nevertheless they show better values of linear and rotational speed than forehands. Decrease net height and court dimensions promote offensive behaviors in young tennis players. This offensive behavior coincides with the recommendations that encourage comprehensive approach to develop an optimal teaching-learning process.
\end{abstract}

Keywords: comprehensive approach, training stage, competitive re-design, smart devices.

Recibido: 05-nov-2019

Aceptado: 11-nov-2019

Publicado: 20-nov-2019

Como citar (apa): Giménez-Egido, J.M., Fernández-García, A.I. \& Castellanos, A. (2020). Análisis del golpeo de fondo en jóvenes jugadores de tenis en una competición modificada mediante sensores inteligentes. JUMP, (1), 39- 44

Como citar (vancouver): Giménez-Egido, J.M., Fernández-García, A.I. \& Castellanos, A. Análisis del golpeo de fondo en jóvenes jugadores de tenis en una competición modificada mediante sensores inteligentes. JUMP. 2020; (1), 39- 44.

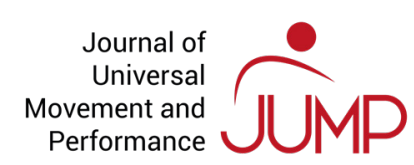




\section{Introducción}

La obtención de información breve, concisa y útil sobre el rendimiento de equipos deportivos y de sus jugadores, ha generado un aumento de la utilización de herramientas tecnológicas que disminuyan el tiempo de análisis por parte de los entrenadores (Fernández-García \& Torres-Luque, 2018; Whiteside, Cant, Connolly, \& Reid, 2017). El propósito principal de su uso, es aumentar el rendimiento de sus jugadores tanto a nivel colectivo como individual, para obtener ventaja sobre sus rivales, al conseguir información concisa y breve que puedan utilizar en tareas y competiciones (FernándezGarcía \& Torres-Luque, 2018). Sin embargo, el uso de estos dispositivos inteligentes con la finalidad de mejorar los procesos de enseñanza-aprendizaje, y/o controlar si se cumple ratios óptimas de oportunidad de aprendizaje en etapas de formación no está tan extendido. Desde la perspectiva de las enseñanzas comprensivas, el uso de dispositivos inteligentes para observar el efecto que tiene la modificación de reglas en tareas y competiciones, tiene un alto valor debido a los cambios provocados a nivel conductual (un pequeño cambio puede generar un gran efecto) (Hastie, Ward, \& Brock, 2017). El objetivo del presente estudio es utilizar un sensor inteligente, para conocer el efecto de una competición modificada (altura de la red= 0,80 $\mathrm{m}$ y dimensiones del campo $=18,00 \times 8,23 \mathrm{~m}$ ) en jugadores de tenis sub-10, obteniendo información referente a: a) número de golpes; b) porcentaje de golpes que impactan en el punto dulce; $c$ ) velocidad de la pelota; y d) revoluciones por minuto (rpm) de la pelota en cada tipo de golpe en el fondo de pista.

\section{Método}

El diseño de estudio es de naturaleza descriptiva y transversal (Ato, López, \& Benavente,
2013). La muestra estaba compuesta por jóvenes jugadores de tenis sub-10 ( $n=20$, edad media $=9.46 \pm 0.66$ años, ) y fueron seleccionados atendiendo a criterios de accesibilidad (Otzen \& Manterola, 2017). El criterio que debían cumplir los participantes era participar en competiciones de un modo asiduo, bajo la reglamentación de la Real Federación Española de Tenis en la etapa Verde, correspondiente al sistema Play and Stay. La toma de datos se realizó mediante el sensor inteligente "Zepp Tennis 2.2.1, Zepp Labs, USA", atendiendo a dos criterios; a) era el único sensor inteligente que se puede utilizar en raquetas junior; y b) ofrecía valores óptimos de fiabilidad en la variable número de golpes y velocidad de bola, por otro lado obtuvo valores moderados en la detección del tipo de efecto (Keaney \& Reid, 2018). Los participantes se distribuyeron en cuatro grupos de cinco jugadores de forma aleatoria, el sistema de enfrentamiento fue de todos contras todo a una sola vuela (round robin). Cada jugador jugó cuatro partidos de un set a cuatro juegos, en caso de empate se disputaría un tie break. La modificación de reglas conllevó la reducción de la altura de la red $(0,80 \mathrm{~m})$ y las dimensiones de la pista $(18 \times 8,23 m)$, el resto de reglas eran iguales al sistema "Verde" de su competición habitual (International Tennis Federation., 2011). Para el control de la calidad del dato se realizó un análisis exploratorio para eliminar posibles valores atípicos, se eliminaron los valores medios que excedían tres veces la desviación estándar, medidas semejantes alas de estudios previos (Muñoz, Gamonales, León, \& Ibáñez, 2018; Shao Liang, Lorenzo Calvo, \& Ángel Gómez Ruano, 2018). Los datos fueron tipificados al no cumplir con los supuestos de normalidad (Field, 2013). El análisis de la varianza se realizó mediante el planteamiento estadístico ANOVA de un factor $(p<0.05)$, además la prueba de homogeneidad de la varianza de Levene indico

Tabla 1. Valores descriptivos (media, desviación estándar, mínimo y máximo) y análisis de la varianza (ANOVA de una factor) del número de golpes por partido según el tipo de golpeo.

\begin{tabular}{|c|c|c|c|c|c|c|}
\hline $\begin{array}{l}\text { Tipo de golpe de } \\
\text { fondo }\end{array}$ & M & $\%$ & SD & Max & Min & Post Hoc \\
\hline Derecha Plana & 36,38 & 42,57 & 20,54 & 94,75 & 8,00 & $\mathrm{DP}>(\mathrm{DL}-\mathrm{DC}-\mathrm{RP}-\mathrm{RL}-\mathrm{RC})$ \\
\hline Derecha Liftada & 16,36 & 19,14 & 10,36 & 40,00 & 4,50 & $\mathrm{DP}>\mathrm{DL}>(\mathrm{RL}-\mathrm{RP})$ \\
\hline Derecha Cortada & 10,74 & 12,57 & 7,44 & 26,25 & 1,75 & $D P>D C$ \\
\hline Revés Plano & 11,16 & 13,06 & 6,00 & 30,25 & 4,33 & $\mathrm{DP}>\mathrm{RP}>(\mathrm{RL}-\mathrm{RC})$ \\
\hline Revés Liftado & 5,27 & 6,17 & 4,23 & 17,50 & 1,00 & $(\mathrm{DP}-\mathrm{DL}-\mathrm{RP})>\mathrm{RL}$ \\
\hline Revés Cortado & 5,19 & 6,07 & 2,13 & 8,50 & 2,00 & $(\mathrm{DP}-\mathrm{DL}-\mathrm{RP})>\mathrm{RC}$ \\
\hline
\end{tabular}

Leyenda: M= media, SD= desviación estándar, Min= valor mínimo y Max= máximo valor encontrado. Abreviaturas golpes de fondo (DP= derecha plana; $\mathrm{DL}=$ derecha liftada, $\mathrm{DC}=$ derecha cortada, $\mathrm{RP}=$ revés plano, $\mathrm{RL}=$ revés liftado, $\mathrm{RC}=$ revés cortado). 
que no se podían asumir varianzas iguales. Por lo tanto, se realizaron comparaciones múltiples Post Hoc utilizando la prueba de Games-Howell como indica la literatura (Pardo \& Ruiz, 2002). La estimación del tamaño del efecto se calculó a través del eta cuadrado parcial (np2): (np2<0.04), mínimo efecto recomendado (0.04 $\leq n \mathrm{p} 2<0.25)$, efecto moderado $(0.25 \leq n p 2<0.64)$, y efecto fuerte (np2 $\geq 0.64)$ (Nakagawa \& Cuthill, 2007). El análisis estadístico se realizó mediante el paquete estadístico "IBM SPSS Statistics 25.0" (IBM Corp., Armonk, NY, United States).

\section{Resultados}

Para cada una de las variables de estudio se ha generado una tabla específica, que permite observar el efecto de la competición modificada en el patrón de golpeo de fondo además de la significación estadística en la comparativa entre golpeos.

En concreto los resultados de la Tabla 1 muestran diferencias significativas entre el número de golpes, en relación a los diferentes tipos de golpeo ( $p=.000 ; F=20.915 ; \eta p 2=.514)$. A nivel genérico es destacable que casi dos tercios de los golpeos se produzcan con la mano dominante (74,28\%), en detrimento de la mano no dominante
(25,30\%). En esta línea, el golpeo plano es la opción más ejecutada por los jugadores duplicando al menos los valores (derecha liftada) de los demás golpeos. En este sentido, el golpe de revés plano duplica los valores del resto de tipo de revés.

El porcentage de golpes que impactan en el punto dulce de la raqueta, muestra que existen diferencias significativas entre los diferentes tipos degolpeo en la Tabla 2 ( $p=.003 ; F=3,958 ; n p 2=.167$ ). Además, se puede observar una diferencia de más de un $20 \%$ entre los golpes de derecha y revés cortados, mientras que en la comparativa entre golpes de derecha y revés con el mismo efecto existe una mayor paridad.

La tabla 3 muestra la velocidad de la pelota trás el golpeo entre los tipos de golpeo de fondo analizados e indica diferencias estadisticamente significativas ( $p=.000 ; F=6,836, \eta p 2=.257$ ). Las velocidades de golpeo más elevadas se producen al utilizar el efecto plano tanto por el lado dominante como no dominante. Por otro lado, se puede observar que la desviación estándar entre en los sujetos en todos los tipos de golpe en el fondo de pista no es superior a $8 \mathrm{~km} / \mathrm{h}$.

En último lugar se puede ver en la tabla 4 la existencia de diferencias estadísticamente significativas, en relación a las rpm que alcanza la pelota entre los diferentes tipos de golpeo $(p=.000$;

Tabla 2. Valores descriptivos (media, desviación estándar, mínimo y máximo) y análisis de la varianza (ANOVA de una factor) de la velocidad de la pelota por partido según el tipo de golpeo.

\begin{tabular}{cccccc}
\hline $\begin{array}{c}\text { Tipo de golpe de } \\
\text { fondo }\end{array}$ & M & SD & Max & Min & Post Hoc \\
\hline Derecha Plana & 71,12 & 10,00 & 422,01 & 87,25 & DP<RC \\
Derecha Liftada & 72,18 & 13,05 & 112,55 & 100,00 & - \\
Derecha Cortada & 62,53 & 19,65 & 57,93 & 97,00 & - \\
Revés Plano & 68,17 & 16,97 & 38,32 & 91,25 & $R P<R C$ \\
Revés Liftado & 79,33 & 19,25 & 16,89 & 100,00 & DP $>$ RL $<$ RC \\
Revés Cortado & 84,65 & 9,83 & 6,03 & 100,00 & $R C>$ (DP-DC-RP) \\
\hline
\end{tabular}

Leyenda: M= media, SD= desviación estándar, Min= valor mínimo y Max= máximo valor encontrado. Abreviaturas golpes de fondo (DP= derecha plana; $\mathrm{DL}=$ derecha liftada, $\mathrm{DC}=$ derecha cortada, $\mathrm{RP}=$ revés plano, $\mathrm{RL}=$ revés liftado, $\mathrm{RC}=$ revés cortado)

Tabla 3. Valores descriptivos (media, desviación estándar, mínimo y máximo) y análisis de la varianza (ANOVA de una factor) de los golpes que impactan en el punto dulce de la raqueta por partido según el tipo de golpeo

\begin{tabular}{cccccc}
\hline $\begin{array}{c}\text { Tipo de golpe de } \\
\text { fondo }\end{array}$ & M & SD & Max & Min & Post Hoc \\
\hline Derecha Plana & 78,38 & 5,15 & 92,50 & 70,25 & DP>(DC-RL-RC) \\
Derecha Liftada & 74,71 & 6,40 & 88,33 & 63,50 & DL>RL \\
Derecha Cortada & 71,24 & 5,81 & 81,00 & 57,33 & DP>DC \\
Revés Plano & 74,96 & 4,53 & 81,75 & 66,50 & $R P>R L$ \\
Revés Liftado & 68,68 & 7,67 & 83,67 & 59,00 & DC-DL-RP>RL \\
Revés Cortado & 70,44 & 4,13 & 76,00 & 61,75 & $D P>R C$ \\
\hline
\end{tabular}

Leyenda: M= media, SD= desviación estándar, Min= valor mínimo y Max= máximo valor encontrado. Abreviaturas golpes de fondo (DP= derecha plana; $\mathrm{DL}=$ derecha liftada, $\mathrm{DC}=$ derecha cortada, $\mathrm{RP}=$ revés plano, $\mathrm{RL}=$ revés liftado, $\mathrm{RC}=$ revés cortado) 
Tabla 4. Valores descriptivos (media, desviación estándar, mínimo y máximo) y análisis de la varianza (ANOVA de una factor) de las revoluciones por minuto de la pelota según el tipo de golpeo

\begin{tabular}{cccccc}
\hline $\begin{array}{c}\text { Tipo de golpe de } \\
\text { fondo }\end{array}$ & M & SD & Max & Min & Post Hoc \\
\hline Derecha Plana & 938,10 & 79,80 & 1115,33 & 785,50 & (DL-DC-RL-RC)>DP \\
Derecha Liftada & 1930,28 & 308,95 & 2610,67 & 1270,00 & DL>(DP-DC-RP-RL) \\
Derecha Cortada & 1528,75 & 214,38 & 2254,50 & 1274,00 & (DL-RC)>DC>(DP-RP) \\
Revés Plano & 1074,22 & 65,34 & 1215,75 & 955,25 & (DL-DC-RL-RC)>RP \\
Revés Liftado & 1541,89 & 225,83 & 1949,50 & 1252,00 & (DL-RC)>RL>(DP-RP) \\
Revés Cortado & 1854,42 & 222,72 & 2202,50 & 1448,67 & $R C>$ (DP-DC-RL-RP)
\end{tabular}

Leyenda: $\mathrm{M}=$ media, $\mathrm{SD}=$ desviación estándar, Min= valor mínimo y Max= máximo valor encontrado. Abreviaturas golpes de fondo (DP= derecha plana; $\mathrm{DL}=$ derecha liftada, $\mathrm{DC}=$ derecha cortada, $\mathrm{RP}=$ revés plano, $\mathrm{RL}=$ revés liftado, $\mathrm{RC}=$ revés cortado)

$F=70,854 ; n p 2=.782)$. Los golpeos de fondo que se realizan con efecto cortado y liftado son los que mayores rpm consiguen imprimir los jugadores a la pelota. No obstante, la derecha liftada es la que alcanza el mayor valor de rpm con más de 400 rpm respecto a la derecha cortada, mientras que el valor más elevado de rpm en el golpeo de revés se produce con el efecto liftado con más 300 rpm respecto al revés liftado.

\section{Discusión}

Este estudio tiene el propósito de usar el sensor inteligente Zepp Tennis, para conocer el efecto de una competición modificada (altura de la red= 0,80 $\mathrm{m}$ y dimensiones del campo $=18,00 \times 8,23 \mathrm{~m}$ ) en jugadores de tenis sub-10, obteniendo información referente al: a) número de golpes; b) porcentaje de golpes que impactan en el punto dulce; c) velocidad de la pelota; y d) rpm de la pelota en cada tipo de golpe en el fondo de pista.

Los resultados de este estudio en líneas generales, denotan un cambió en el patrón conductual de los jugadores, que concuerda con las premisas de las enseñanzas comprensivas en la creación de contextos ecológicos que fomenten la oportunidad de aprendizaje en jóvenes jugadores. Este cambio conductual se pueden observar con el aumento del número de golpeos planos, los cuales se asocian con una predisposición ofensiva, al reducir el tiempo de reacción del adversario al aumentar la velocidad de bola, al igual que en otros estudios (Limpens, Buszard, Shoemaker, Savelsbergh, \& Reid, 2018; Timmerman et al., 2015). En relación a la idea anterior, la competición actual en etapa sub-10 (verde) fomenta el juego liftado con bote alto, al forzar a su rival a golpear la pelota por encima de su hombro (búsqueda del fallo del adversario). Diversos estudios asocian esa conducta a un patrón de juego defensivo y poco creativo (Bayer, Ebert, \& Leser, 2017; Schmidhofer, Leser, \& Ebert, 2014). Estos datos muestran que la reducción de la altura de la red y las dimensiones de la pista se adaptan mejor a las características de los jugadores en etapa formativa, al facilitar el juego de ataque como se promulgan en las nuevas perspectivas ecológico-dinámicas (Chang, Chow, Button, \& Tan, 2017; Renshaw \& Chow, 2019).

Desde el punto de vista del fomento de la variabilidad de juego, la participación en esta competición no ha obtenido un número de golpeos semejantes entre todos los tipos de golpe. Aunque si se observa como determinados tipos de golpeo si son fomentadas por entrenadores en el diseño de tareas podrían ser incluidas en el bagaje técnico-táctico de los jóvenes jugadores en competición, en concreto se podría obtener éxito el fomento de: a) golpe cortado de revés por ser el segundo valor mayor de rpm en el golpeo; y b) revés plano debido a que es el segundo tipo de golpe que genera mayor velocidad de bola. En definitiva existen golpeos ejecutados por el lado no dominante que podían generar rendimiento, si se utilizarán con mayor asiduidad, como se refleja en el estudio de Farrow \& Reid (2010) en jóvenes jugadores y en alto rendimiento por Blanca-Torres, Fernández-García, \& Torres-Luque, (2019).

Por último, la competición modificada ayuda a equiparar las características físicas de los jugadores, debido a que los valores de velocidad de bola inter-sujetos (desviación estándar) son semejantes, no actuando la maduración físicobiológica como un factor limitante del aprendizaje. Este tipo de competiciones ayudan a equiparar las diferencias físicas en estas edades (la fuerza de golpeo se asocia a la velocidad), fomentando una competición para todos, que mejore la toma de decisiones y el uso de conductas creativas para obtener rendimiento (Araujo, Davids, \& Hristovski, 2006; McCarthy, Bergholz, \& Bartlett, 2016). 
El uso y manejo de dispositivos inteligentes para obtener información sobre el efecto de tareas y competiciones modificadas, es una realidad latente que los entrenadores deben dominar, para mejorar los procesos de enseñanza-aprendizaje (Fister, Fister, \& Fister, 2019; Memmert, Lemmink, \& Sampaio, 2017). Los resultados de este estudio muestran como este tipo de competiciones se ajustan a las necesidades de los jóvenes jugadores, promoviendo conductas que mejoran la oportunidad de aprendizaje (Chow, Davids, Button, \& Renshaw, 2016; García-Angulo, Giménez-Egido, García-Angulo, \& Ortega-Toro, 2019).

Como todo proceso de investigación, el efecto generado por la modificación de elementos que median en el comportamiento humano, se deben tomar con cautela debido a dos aspectos principalmente: a) se debe replicar este tipo de competiciones modificadas en muestras con semejantes o diferentes características, con este u otros dispositivos para extraer resultados estables (Gonçalves, Klering, Aires, \& Balbinotti, 2016); y b) la falta de experiencia de los jugadores en este tipo de competiciones puede limitar el efecto de la misma, no observándose todo el potencial de la competición propuesta.

\section{Conclusiones}

Cuatro conclusiones se pueden extraer de la presente investigación: a) el uso de dispositivos inteligentes, ayuda al conocimiento sobre el efecto de las modificaciones de reglas y equipamientos deportivos; b) los jugadores adoptan una conducta ofensiva al facilitarse el golpeo plano ante la reducción de la altura de la red y las dimensiones de la pista; c) existen carencias formativas en el uso del golpeo de revés, aunque tengan valores similares e incluso superiores de velocidad lineal y de giro respecto a los golpeos de derecha; y d) las modificaciones planteadas equiparan las capacidades físicas de los jugadores, pudiendo fomentar acciones técnico-tácticas creativas para obtener éxito.

\section{Aplicaciones Prácticas}

Los resultados extraídos carecen de valor si no sirven para ayudar a los entrenadores en el proceso de formación de jóvenes jugadores, de ahí que sea necesario establecer una aplicabilidad práctica, enfocada tanto al diseño de tareas como de competiciones. Quizás una de las cuestiones que no se han llegado a cubrir con esta competición modificada es el concepto de variabilidad en la práctica. Esta situación se debeal uso predominante del golpe de derecha plano por encima de los otros tipos de golpeo, siendo una posible causa la falta de experiencia en este tipo de competiciones un factor limitante de aprendizaje. De este modo, los entrenadores deben promover competiciones que ayuden a la aparición todos los tipos de golpeo, un ejemplo sería modificar las normas de puntuación ya sea en tareas o competiciones del siguiente modo:

- Los puntos ganados tras golpe de revés tendrán un valor doble.

- Suma de juegos extra en función del uso del golpeo de revés durante toda la jornada de competición, es decir el sumatorio de todos los puntos ganados con el golpe de revés obtendrá los siguientes juegos extra; de 0 a 5 puntos con el revés $=1$ juego extra, de 5 a 10=2 juegos extra, de 10 a 15= 3 juegos extra, por encima de 15 puntos= cuatro juegos extra. Ganará el torneo el jugador que más juegos acumule.

- La puntuación será doble si durante el punto se realizan tres tipos diferente de golpeo por parte del ganador del punto, un ejemplo seria la siguiente secuencia; revés plano, derecha cortada y derecha liftada.

- Se conseguirá una puntuación doble si el golpeo con el que se finaliza el punto es un golpeo de revés plano o cortado, al entender la velocidad lineal y angular como factor de rendimiento respectivamente (ver Tablas 3 y 4).

Entendiendo la competición como un factor muy influyente en el proceso de enseñanzaaprendizaje, sería beneficioso construir torneos donde los jugadores disputen partidos con diferentes formatos en una misma jornada (Farias, Harvey, Hastie, \& Mesquita, 2019). La finalidad sería crear experiencias basadas en la teoría de la "repetición sin repetición", que implementen la oportunidad de aprendizaje trabajando la creatividad, la variabilidad y la cantidad de práctica. 


\section{Financiación}

El presente trabajo ha sido financiado parcialmente gracias a las ayudas concedidas por el Ministerio de Economía y competitividad, España (DEP2016-76873-P) así como por el Ministerio de Educación, cultura y deportes (PRX18/00217)

\section{Referencias}

Araujo, D., Davids, K. W. \& Hristovski, R. (2006). Ecological Dynamics of Decision-Making. Psychology of Sport and Exercise, 7, 653676.

Ato, M., López, J. J. \& Benavente, A. (2013). Un sistema de clasificación de los diseños de investigación en psicología. Anales de Psicologia, 29(3), 1038-1059.

Bayer, D., Ebert, M. \& Leser, R. (2017). A comparison of the playing structure in elite kids tennis on two different scaled courts. International Journal of Performance Analysis in Sport, 17(12), 34-43.

Blanca-Torres, J. C., Fernández-García, Á. I. \& Torres-Luque, G. (2019). Influencia de la categoría y el genero en variables temporales en el tenis individual de élite. Journal of Sport and Health Research, 11(1), 69-78. http://www.journalshr.com/ papers/Vol\%2011_N\%201/JSHR\%20V11_1_6.pdf

Chang, M. Y. L., Chow, J. Y., Button, C. \& Tan, C. W. K. (2017). Nonlinear pedagogy and its role in encouraging 21st century competencies through physical education: A Singapore experience. Source Asia Pacific Journal of Education, 37(4), 483-499.

Chow, J. Y., Davids, K., Button, C. \& Renshaw, I. (2016). Nonlinear Pedagogy in Skill Acquisition. In Nonlinear Pedagogy in Skill Acquisition

Farias, C. F. G., Harvey, S., Hastie, P. A. \& Mesquita, I. M. R. (2019). Effects of situational constraints on students' game-play development over three consecutive Sport Education seasons of invasion games. Physical Education and Sport Pedagogy, $0(0), 1-20$.

Farrow, D. \& Reid, M. (2010). The effect of equipment scaling on the skill acquisition of beginning tennis players. Journal of Sports Sciences, 28(7), 723-732.

Fernández-García, A. \& Torres-Luque, G. (2018). Intelligent devices for tennis rackets. ITFCoaching \& Sport Science Review, 26(75), 5-7.

Field, A. (2013). Discovering statistics using IBM SPSS statistics (Michael Carmichael Development, ed.).

Fister, I., Fister, I. \& Fister, D. (2019). Design and Implementation of an Artificial Sports Trainer. In Computational Intelligence in Sports. Adaptation, Learning, and Optimization (Vol. 22, pp. 121-135).

García-Angulo, A., Giménez-Egido, J. M., García-Angulo, F. J. \& Ortega-Toro, E. (2019). Revisión de los reglamentos de balonmano en categorías de formación en españa. E-Balonmano.Com: Journal of Sport Science, 15(1), 9-22.

Gonçalves, G. H. T., Klering, R. T., Aires, H. \& Balbinotti, C. A. A. (2016). Contribuições da competição de tênis na educação e formação de crianças. Journal of Physical Education, 27(1), 2738.

Hastie, P. A., Ward, J. K. \& Brock, S. J. (2017). Effect of graded competition on student opportunities for participation and success rates during a season of Sport Education. Physical Education and Sport Pedagogy, 22(3), 316-327.

International Tennis Federation. (2011). Tennis 10s: The ITF guide to organising 10 and under competition (England). London.

Keaney, E. M. \& Reid, M. (2018). Quantifying hitting activity in tennis with racket sensors: new dawn or false dawn? Sports Biomechanics, 00(00), 1-9.

Limpens, V., Buszard, T., Shoemaker, E., Savelsbergh, G. J. P. \& Reid, M. (2018). Scaling Constraints in Junior Tennis: The Influence of Net Height on Skilled Players' Match-Play Performance. Research Quarterly for Exercise and Sport, 89(1), 1-10.

McCarthy, J., Bergholz, L. \& Bartlett, M. (2016). Re-designing Youth Sport: Change the Game.

Memmert, D., Lemmink, K. A. P. M. \& Sampaio, J. (2017). Current Approaches to Tactical Performance Analyses in Soccer Using Position Data. 47, 1-10.

Muñoz, J. ., Gamonales, J. M., León, K. \& Ibáñez, S. J. (2018). Formación de codificadores y fiabilidad de registros. Una aplicación Global. Revista Internacional de Medicina y Ciencias de La Actividad Física y El Deporte., 18(72).

Nakagawa, S. \& Cuthill, I. C. (2007). Effect size, confidence interval and statistical significance: a practical guide for biologists. Biol. Rev, 82, 591-605.

Otzen, T. \& Manterola, C. (2017). Técnicas de Muestreo sobre una Población a Estudio Sampling Techniques on a Population Study. Int. J. Morphol, 35(1), 227-232.

Pardo. A, \& Ruiz, M. A. (2002). Análisis de varianza de un factor: El procedimiento ANOVA de un factor. In SPSS 17: Guia para el analisis de datos (Mc Graw Hi, pp. 251-256).

Renshaw, I. \& Chow, J. Y. (2019). A constraint-led approach to sport and physical education pedagogy. Physical Education and Sport Pedagogy, 24(2), 103-116.

Schmidhofer, S., Leser, R. \& Ebert, M. (2014). A comparison between the structure in elite tennis and kids tennis on scaled courts (Tennis 10s). International Journal of Performance Analysis in Sport, 14(3), 829-840.

Shao Liang, Z., Lorenzo Calvo, A. \& Ángel Gómez Ruano, M. (2018). Clustering performances in the NBA according to players' anthropometric attributes and playing experience. Journal of Sports Sciences, 36(22), 2511-2520.

Timmerman, E., De Water, J., Kachel, K., Reid, M., Farrow, D. \& Savelsbergh, G. (2015). The effect of equipment scaling on children's sport performance: the case for tennis. Journal of Sports Sciences, 33(10), 1093-1100.

Whiteside, D., Cant, O., Connolly, M. \& Reid, M. (2017). Monitoring Hitting Load in Tennis Using Inertial Sensors and Machine Learning. International Journal of Sports Physiology and Performance, 12, 1212-1217. 